\title{
Granular flow fluctuations in a conical hopper
}

\author{
Guilhem Mollon ${ }^{1, *}$, and Jidong Zhao ${ }^{2}$ \\ ${ }^{1}$ LaMCoS, INSA Lyon, UMR 5259, Université de Lyon, CNRS, 69621 Villeurbanne Cedex, France \\ ${ }^{2}$ Department of Civil and Environmental Engineering, Hong Kong University of Science and Technology, Clearwater Bay, Hong Kong
}

\begin{abstract}
In this article, we present simulations of 3D hopper flows through conical hoppers, with a focus on time fluctuations of flow-related quantities (velocity, coordination number and solid fraction). Dedicated post-processing techniques are used to obtain local and yet statistically meaningful measurements of these quantities. Simulations show that the fluctuations that were already observed in 2D hopper flows are still present in 3D. A parametric study also demonstrates that the frequency of the fluctuations is strongly related to the stiffness parameter of the contact law used in the simulations.
\end{abstract}

\section{Introduction}

Fluctuations of granular flows in conical hoppers have been reported both experimentally [1-3] and numerically [4-6] in the granular science literature. They are often attributed to intermittent arch networks above the outlet of hoppers which trigger high-frequency instationarities of the flow [6], or stress-related static avalanches [1]. In a previous work in 2D [3], we demonstrated that these instationarities take the form of organized waves which propagate upstream in the hopper. However, conclusions drawn in 2D do not necessarily apply in 3D, since the stability, strength and dynamics of force chains are rather different in both topologies. The 3D case is investigated here in a similar DEM framework, in order to determine (i) if these organized instationarities do exist in a 3D topology and (ii) if they do behave as acceleration waves propagating upwards. Besides, the results of the first stage of a comprehensive parametric study are presented and their implications on discrete modelling of granular flows are detailed.

\section{Simulations}

In order to study flow fluctuations, simulations are driven in the standard DEM framework. Spherical particles are used, with a unit density, a unit average diameter (this diameter is used all along this paper as a unit for distances) and a moderate polydispersity (i.e. a coefficient of variation of the grains volumes equal to 0.4). Contacts between grains and with walls are penalized in the normal and tangential direction by a contact stiffness $K_{n}$. Normal kinetic energy is damped by a viscous dashpot with a dissipation parameter of 0.2 (with the classical definition, see e.g. [5]). In the tangential direction, Coulomb friction is considered, with a coefficient of 0.4 . The equations of motion are solved with a velocity-Verlet explicit scheme. Since linear contact laws (in displacement and velocity) are used, classical scaling rules make it possible to generalize these results to other geometries (in contrast with the remarks of [7], into which nonlinear stiffness and normal damping are used). The hopper has a conical shape, with an opening angle equal to $70^{\circ}$. Its outlet is a circle of diameter 10. It is filled with approximately 50,000 grains which are deposited under gravity. The outlet is then opened a $\mathrm{t}=0$, triggering the flow through the hopper.

Fig. 1 shows the proportion of material passed through the outlet along time, for various values of the contact stiffness ranging from $\mathrm{K}_{\mathrm{n}}=10^{4}$ to $10^{8}$. It appears from this figure that the flow rate is constant (i.e. independent from the height of material in the hopper) and does not depend on the contact stiffness.

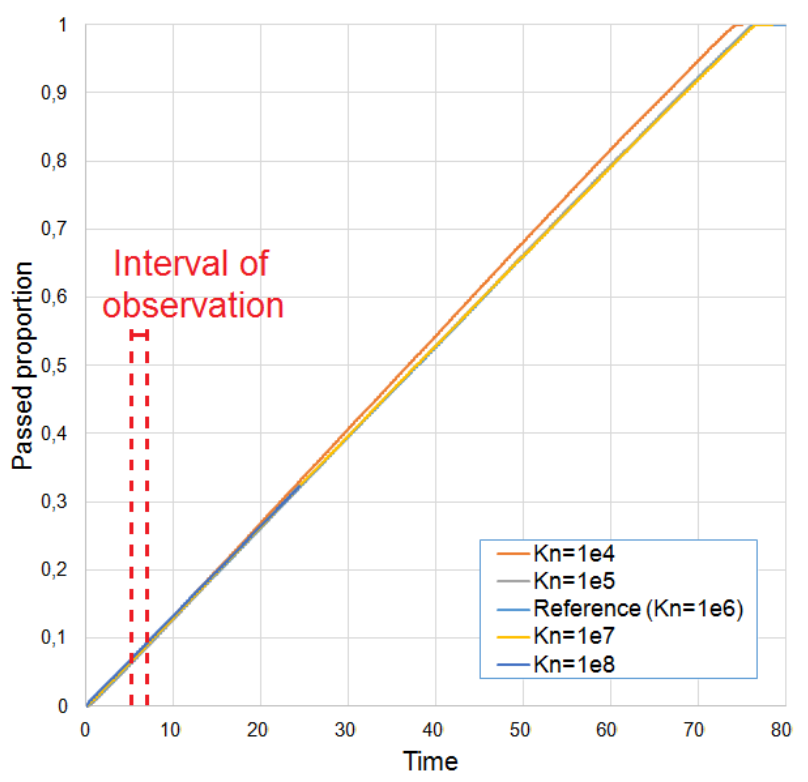

Fig. 1. Proportion of material passed through the hopper in time, for different values of the contact stiffness.

\footnotetext{
Corresponding author: guilhem.mollon@gmail.com
} 


\section{Fluctuations}

In the remainder of this article, attention is paid to a narrow time period (between instants $\mathrm{t}=5$ and $\mathrm{t}=7$ ), illustrated in Fig. 1 and called hereafter the "interval of observation". Fig. 2 shows two snapshots of the velocity fields (i.e. velocity norms of each grain), at two close instants in this interval, for the simulation corresponding to $K_{n}=10^{6}$. From these snapshots, it clearly appears that the velocity field is not stationary.

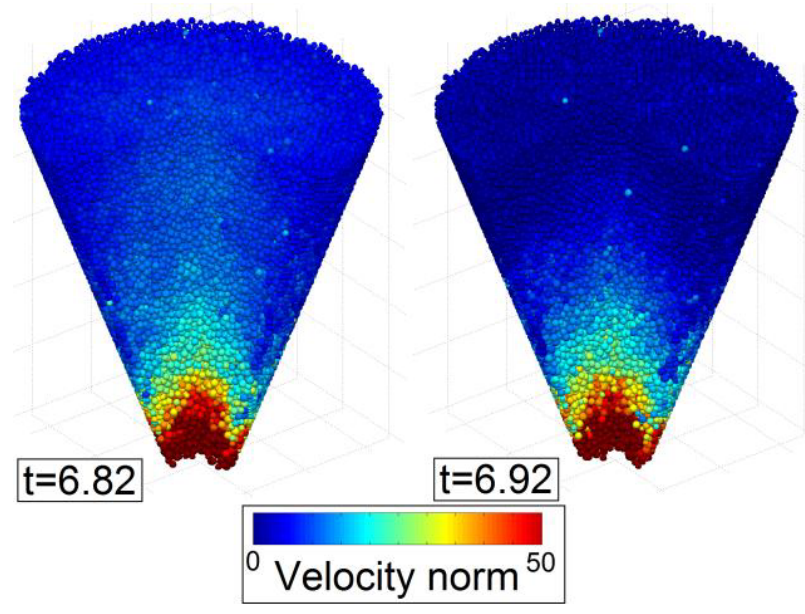

Fig. 2. Time fluctuations of the velocity field.

In order to characterize in a more quantitative way these fluctuations, appropriate data processing is needed. Indeed, some relevant data are attached to grains (e.g. velocity and coordination number, i.e. number of contacts per grain), while some others are attached to collections of grains (e.g. solid fraction). In any case, all these quantities do follow the grains in their motion, as always in a Lagrangian framework. Conversely, a proper statistical analysis requires an Eulerian description, where quantities are attached to fixed spatial locations. For this purpose, two different approaches are used:

-grain-related quantities (velocity and coordination number) are interpolated at fixed locations between grains using moving least squares, in a spherical domain with a radius equal to 5 distance units around the desired location.

-A local solid fraction at a given location $(x, y, z)$ of space is computed by a weighted averaging technique which complies with the principle of mass conservation:

$$
F_{s}(x, y, z)=\left(\pi d^{2}\right)^{3 / 2} \Sigma V_{i} \mathrm{e}^{-\mathrm{ri}(\mathrm{x}, \mathrm{y}, \mathrm{z}) / \mathrm{d}}
$$

In this expression $V_{i}$ corresponds to the volume of the grain $i, r_{i}(x, y, z)$ corresponds to the distance from the centre of this grain to the location $(x, y, z)$, and the summation is executed on all the grains located in a sphere of radius $d=8$ around this location.

These post-processing techniques are used to analyse the evolutions of velocity, coordination number and solid fraction along a vertical profile located on the axis of revolution of the hopper. This is performed every 0.002 time units during the interval of observation, in order to draw an accurate picture of the fluctuations of the hopper flow. Acquired data are summarized in Fig. 3.

In this figure, it clearly appears that the fields of velocity, coordination number and solid fractions are submitted to organized variations, albeit to various extents. Velocity and coordination number seem to obey to unpredictable fluctuations in the lower part of the hopper (i.e. below a height of 5 average diameters for coordination number, and 15 for velocity) and to evolve towards organized and periodic patterns above this height. Regarding velocity, the intensity of these fluctuations is rather limited, while the coordination number fluctuates more strongly, especially in the upper part of the flow.

Solid fraction, in contrast, shows extremely faint fluctuations (Fig. 3c.-left), with an interplay between two patterns (Fig. 3c.-right): some slow variations related to the downward motion of organized clusters of grains, and some rapid fluctuations with the same period as those of velocity and coordination number.

\section{Influence of contact stiffness}

In many mechanical systems, especially in continuum and structural mechanics, natural oscillations are related to the dynamic interplay between density and stiffness (like for example in the spring-mass classical system). In a granular system composed of rigid grains, the only stiffness (i.e. ratio between a force and a displacement) in the system is the contact stiffness. This parameter is well-known for controlling the local oscillations, because the period of the most frequent eigenmode of the system drives the time step used for resolution, and hence the simulation cost.

To investigate the influence of the contact stiffness on the fluctuations, this parameter is varied from $10^{4}$ to $10^{8}$, i.e. over 4 decades. Fig. 4 shows two space-time diagrams corresponding to the evolution of the coordination number on the vertical axis of revolution of the hopper, for values of the stiffness equal to $10^{5}$ and $10^{7}$.

It appears that the contact stiffness has a large influence on the flow fluctuations. When the stiffness is small (i.e. soft contacts), the fluctuations occur at a lower frequency, and the velocity of propagation of the upstream "waves" (i.e. the slope of the patterns observed in the space-time diagram) is quite low. On the contrary, when the stiffness is high (i.e. hard contacts), fluctuations are very frequent, and there is no noticeable upwards propagation of the pattern (or this propagation occurs too fast to be observed at this sampling rate and the fluctuations appear synchronized in space). In both cases, the amplitudes of the variations seem to be rather similar. 

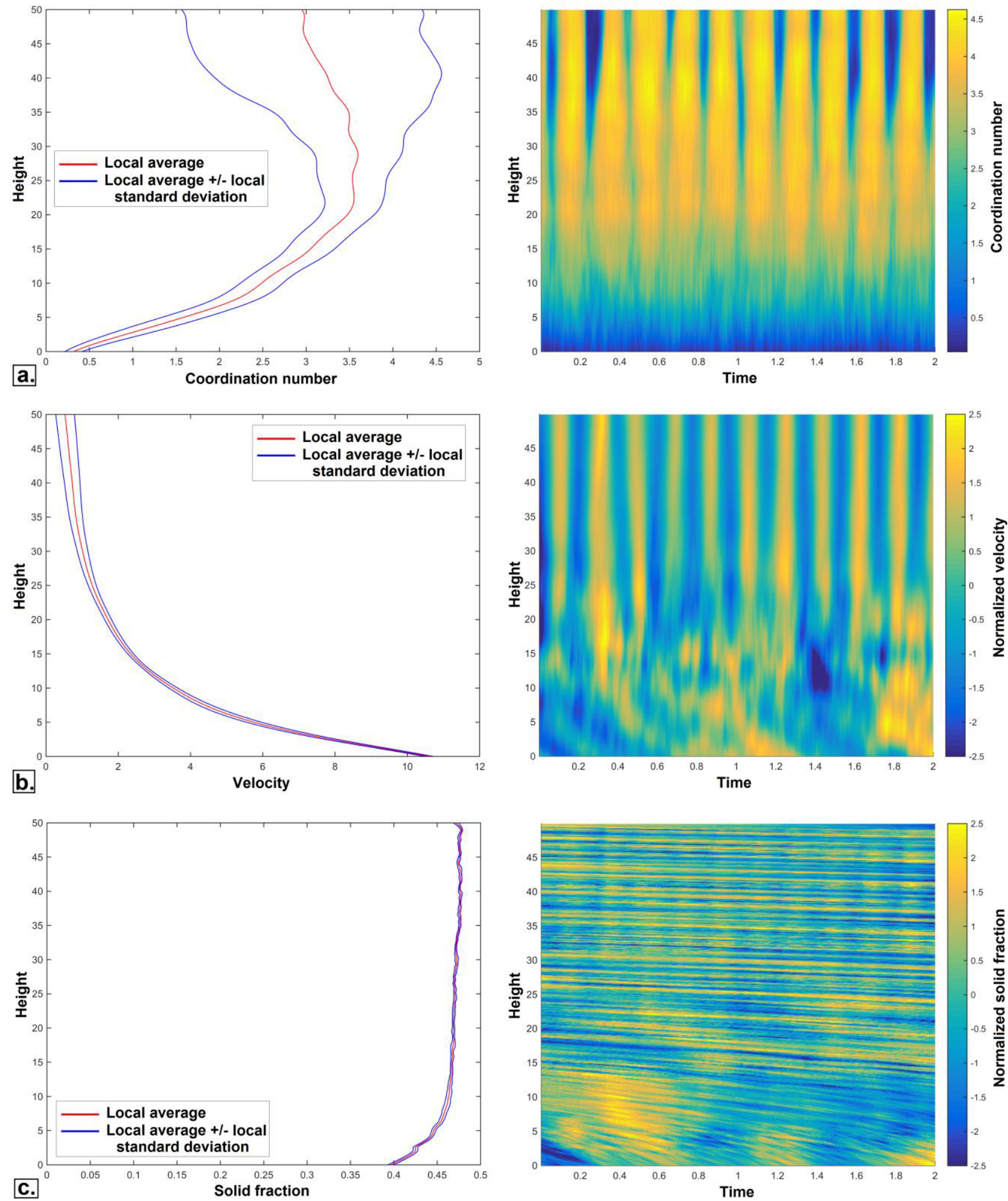

Fig. 3. Statistics of several quantities during the interval of observation. Left: Time-averaged values (plus and minus one time-related standard deviation) as a function of the vertical position ; Right: Space-time diagrams ; a. Coordination number ; b. Velocity ; c. Solid fraction. For b. and c., quantities were normalized in the space-time diagrams to enhance the visibility of fluctuations: to each local instantaneous value, the local time-average was subtracted, and the result was divided by the local time-related standard deviation. 

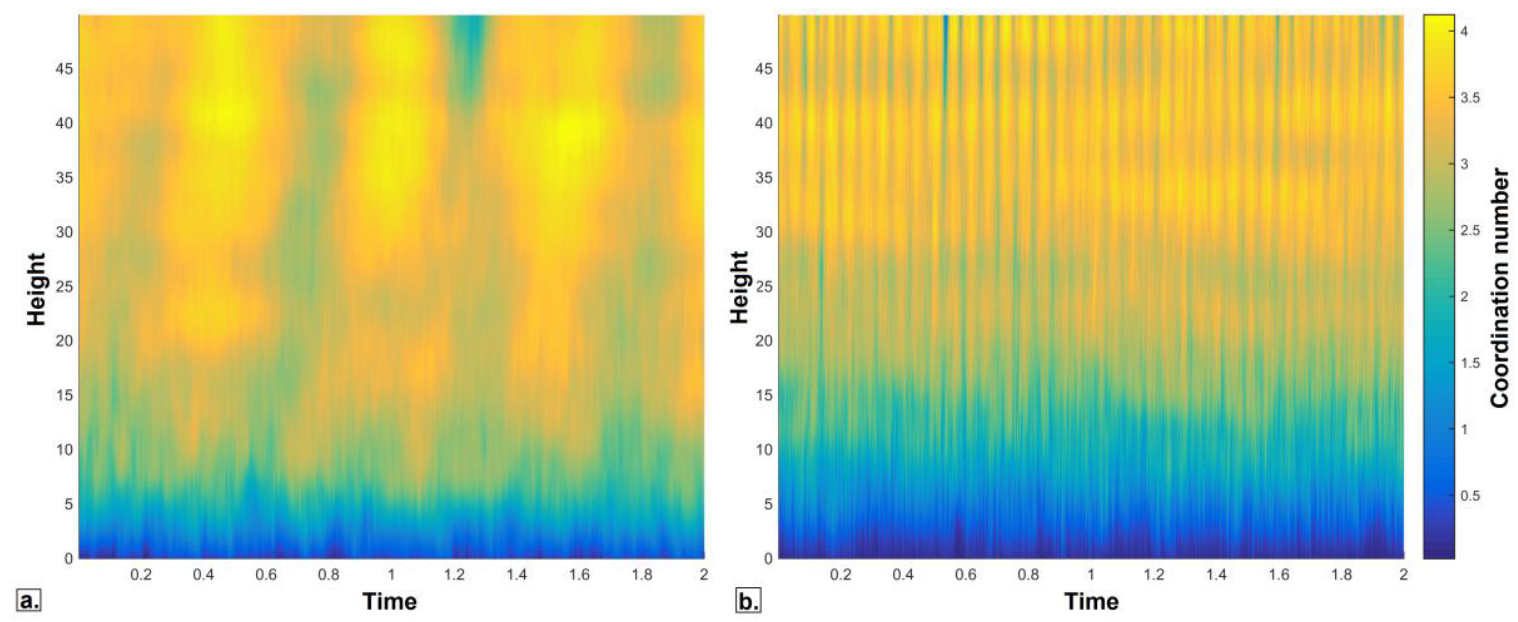

Fig. 4. Space-time diagrams of the coordination number for two different values of the contact stiffness. a. $\mathrm{Kn}=10^{5} ; \mathrm{b} . \mathrm{Kn}=10^{7}$.

Fig. 5 provides the evolution of the main frequency of the fluctuations (obtained by computing the autocorrelation function of the time evolution of the coordination number at a height of 40 average diameters and by running a peak search) as a function of the contact stiffness. This figure clearly shows that the period is proportional to the inverse square root of the contact stiffness.

This behaviour is typical of eigenmodes in structural mechanics, but is rather surprising in the framework of granular mechanics because the fluctuations observed here are not local, but are at the scale of the system. Besides, this result seems to indicate that the same eigenmode is activated in all the five simulations, whatever the value of the contact stiffness. The dynamic mechanisms leading this system to evolve towards established excitations of a given mode of vibration are yet to be understood.

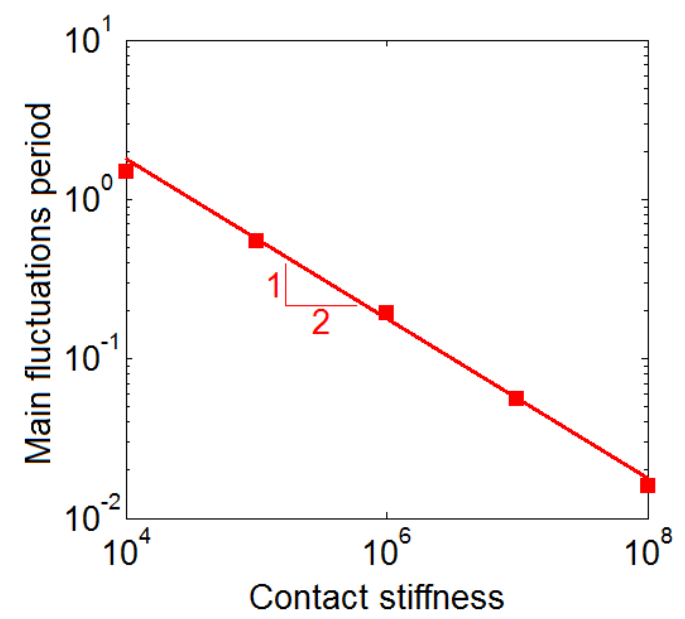

Fig. 5. Influence of the contact stiffness on the main period of the fluctuations.

\section{Conclusion and prospects}

The preliminary results reported in this communication indicate that granular flows through 3D conical hoppers are spontaneously submitted to organized and periodic fluctuations, in a way similar to the $2 \mathrm{D}$ case. It appears that the main period of these fluctuations scales with the inverse square root of the contact stiffness.

This finding gives rise to several questions, among which an important one is related to the way contact stiffness is considered in a modelling framework. Indeed, this parameter may be either considered as a physical parameter (accounting for the deformability of the grains, in which case it is related to their Young modulus and Poisson ratio) or as a numerical parameter (as a penalization of the contact interpenetration, like in FEM contacts). The latter situation is more common, because (i) accounting for a realistic stiffness of the grains usually leads to very stiff contacts and to prohibitive computational costs, and (ii) it is often found that this parameter has no influence on the physical phenomena of interest for the modeller (see e.g. Fig. 1).

Based on the results of the present paper, it appears that, if fluctuations are the actual phenomenon that the modeller wishes to investigate, then the contact stiffness should be considered as a physical parameter and chosen appropriately to reflect the actual stiffness of the grains.

\section{References}

1. P. Claudin, J.P. Bouchaud, PRL 78(2), 231-234 (1997)

2. E. Gardel, E. Seitaridou, K. Facto, E. Keene, K. Hattam, N. Easwar, N. Menon, Phil. Trans.. Roy. Soc. A 367, 5109-5121 (2009)

3. A. Garcimartin, I. Zuriguel, A. Janda, D. Maza, PRE 84, 031309 (2011)

4. P.W. Cleary, M.L Sawley, Appl. Math. Model. 26, 89-111 (2002)

5. R. Arévalo, A. Garcimatin, D. Maza, Eur. Phys J. E 23, 191-198 (2007)

6. G. Mollon, J. Zhao, Gran. Mat. 15(6), 827-840 (2013)

7. T. Pöschel, C. Salueña, T. Schwager, PRE 64, 011308 (2001) 